\title{
Neuropeptides and peptide hormones in syncope and orthostatic intolerance
}

\author{
Balaji Krishnan, David G. Benditt \\ Cardiac Arrhythmia/Syncope Center (Syncope Clinic), Cardiovascular Division, \\ University of Minnesota Medical School, Minneapolis, Minnesota, United States
}

\begin{abstract}
Syncope and orthostatic intolerance (OI) are common clinical syndromes often requiring medical attention. The former is defined as transient loss of consciousness and postural tone due to self-limited cerebral hypo-perfusion, while the latter consists of inappropriate cardiovascular responses to upright posture such as occur with orthostatic hypotension $(\mathrm{OH})$ or postural orthostatic tachycardia syndrome. The most frequent causes of syncope and OI are conditions that temporarily disrupt essential moment-to-moment interaction between the autonomic nervous system and cardiovascular system. In this regard, many neuropeptides (NPs) or peptide hormones $(P H)$ exert cardio-active effects that might contribute to the pathophysiology of certain forms of syncope or OI. To date, the principal peptides that have been studied in this context are: atrial and B-type-neuropeptides, adrenomedullin, endothelin-1 (ET-1), galanin, and vasopressin. While definitive conclusions cannot yet be drawn, the intrinsic vasoconstrictor ET-1 appears to be elevated in $\mathrm{OH}$, presumably to compensate for vasodilation and hypotension of other etiologies. As such elevated ET-1 may become a marker for OH. Further, elevated NT-proBNP may play a role in causing vasodilation and hypotension in some forms of $\mathrm{OH}$ of previously unknown cause, and may be a marker in other patients of a cardiovascular cause of syncope and OI. In the end, the study of the role of NPs and PHs in syncope and OI syndromes is at an early stage, and considerable further future effort is needed. (Cardiol J 2014; 21, 6: 591-600)
\end{abstract}

Key words: neuropeptides, peptide hormones, syncope, orthostatic intolerance, autonomic nervous system, natriuretic peptides

\section{Introduction}

Syncope and orthostatic intolerance (OI) are common clinical conditions with many possible etiologies (Fig. 1) [1, 2]. By far the most frequent causes are conditions that transiently disrupt essential moment-to-moment interaction between the autonomic nervous system (ANS) and the cardiovascular system (CVS); others include:

- Adverse effects of a wide variety of drugs that operate through modification of CVS or nervous system responsiveness, or volume status;
- Inadequate circulating volume such as may occur in older individuals exposed to hot dry environments or who have inadequate thirst drive;

- Complications of cardiovascular disease, particularly cardiac arrhythmias;

- Chronic diseases of the nervous system that result in hypotension during orthostatic stress or physical exertion.

In health, ANS and CVS work together to assure appropriate cerebral perfusion [3]. If this collaboration is disturbed, even briefly, cerebrovascular blood flow may be compromised sufficiently

Address for correspondence: David G. Benditt, MD, FACC, FHRS, FRCPC, FESC, Cardiac Arrhythmia Center, University of Minnesota Medical School, Mail Code 508, 420 Delaware St SE, Minneapolis, MN, 55455, USA, tel: 612 6254401, fax: 612 6244937, e-mail: bendi001@umn.edu

Received: 19.08.2014

Accepted: 07.09.2014 


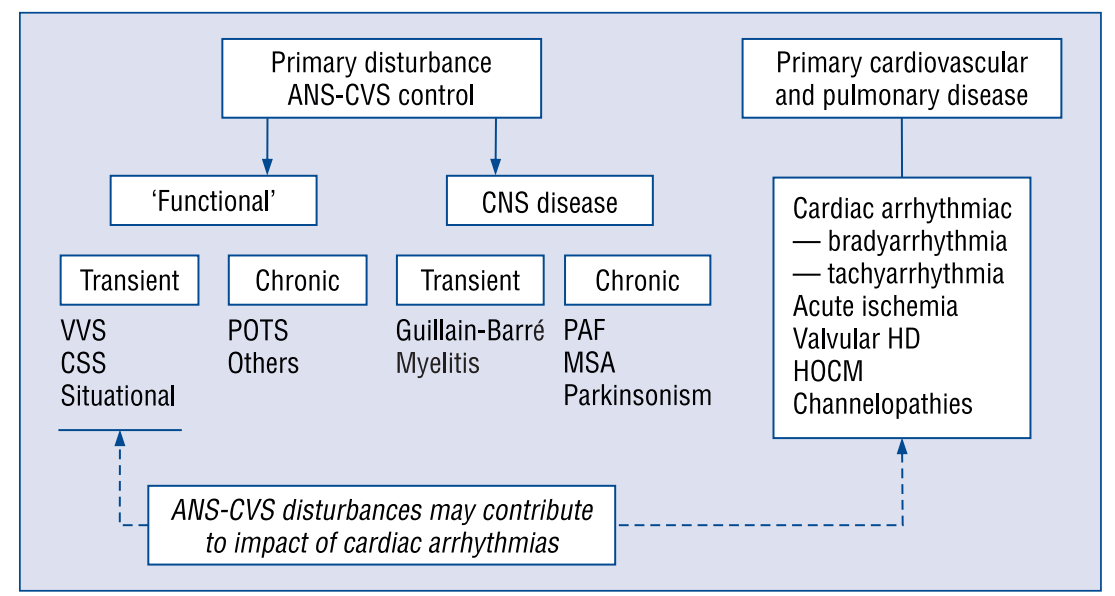

Figure 1. Classification and principal causes of syncope and orthostatic intolerance; ANS - autonomic nervous system, CNS - central nervous system; CSS — carotid sinus syndrome; CVS — cardiovascular system; HD — heart disease; HOCM — hypertrophic cardiomyopathy; MSA — multiple system atrophy; PAF — pure autonomic failure; POTS - postural orthostatic tachycardia syndrome; VVS — vasovagal syncope.

to cause complete or near loss of consciousness (i.e., syncope, near-syncope), or other frequent but less well-defined symptoms such as 'dizziness' and 'lightheadedness'. For example, even temporary inappropriate partitioning of circulatory volumes among various vascular beds may result in excessive pooling of blood in the sub-diaphragmatic circulation; the result may be cerebral hypo-perfusion during upright posture (i.e., orthostatic intolerance) and it may be accompanied by syncope or near syncope.

Since most clinical disturbances of ANS-CVS control occur in the absence of evident structural ANS or CVS disease and have resolved before medical attention is sought (e.g., reflex faints such as vasovagal syncope), establishing the specific responsible etiology and pathophysiology of the patient's symptoms is often challenging. On the other hand, understanding of the specific cause and the pathophysiology provides the necessary foundation needed to devise effective therapy for prevention of future attacks. Consequently, there has been growing interest in identifying biochemical markers that may provide clues to the underlying diagnosis of a recent but resolved spell, or perhaps might even contribute directly to the underlying problem. In this regard, certain neuropeptides (NPs) or peptide hormones (PHs) that are released into the circulation under conditions of stress have attracted particular interest (Tables 1,2).

It is recognized that studies of the potential value of NPs and PHs in pinpointing likely causes of syncope and/or OI in given patients, and in providing greater insight into the pathophysiology of these conditions has only just begun. Nonetheless, the goal of this review is to summarize current understanding and identify topics that may merit future investigation. For convenience, the abbreviation NP will be used to indicate both NPs and PHs.

\section{Syncope and OI}

As noted above, while syncope and OI have many potential causes (Fig. 1), those conditions that result in a 'functional' disturbance of ANS-CVS control are by far the most common [1]. In most instances the functional abnormality is transient and unpredictable in nature, and triggers syncope or near-syncope (or perhaps 'lightheadedness') quite unexpectedly; vasovagal syncope and situational reflex faints are the best examples. However, chronic functional disturbances of ANS-CVS control may also occur, leading to long-standing disability. The most widely recognized example of this is the multi-faceted postural orthostatic tachycardia syndrome (POTS), but other less well recognized conditions almost certainly exist $[4,5]$. On the other hand, not all forms of 'functional' reflex syncope conditions fit neatly into the 'transient' or 'chronic' categories. For instance, carotid sinus syndrome (CSS) is intermittent and is similarly unpredictable in terms of the triggering of a symptomatic neural reflex; however, CSS is usually associated with chronic underlying predisposing factors such as carotid vascular atheromatous disease or neck muscle (particularly sternocleidomastoid) neural deafferentation. The latter may adversely 
Table 1. Main sites of origin of neuropeptides/peptide hormones assessed to date for syncope and orthostatic intolerance.

\begin{tabular}{lccccc}
\hline Neuropeptide & $\begin{array}{c}\text { Brain and/or } \\
\text { nerves }\end{array}$ & Heart & Vessels & $\begin{array}{c}\text { Gastrointestinal } \\
\text { tract }\end{array}$ & Kidneys \\
\hline B-type natriuretic peptide & $\checkmark$ & $\checkmark$ & & \\
Atrial natriuretic peptide & & $\checkmark$ & & \\
Adrenomedullin & & $\checkmark$ & $\checkmark$ & \\
Endothelin (1-3) & $\checkmark$ & & $\checkmark$ & $\checkmark$ \\
Galanin & $\checkmark$ & & $\checkmark$ & $\checkmark$ \\
Vasoactive intestinal peptide & $\checkmark$ & & & \\
Vasopressin & & & \\
\hline
\end{tabular}

Table 2. Primary actions of neuropeptides/peptide hormones.

\begin{tabular}{lcccc}
\hline Neuropeptide & Vasodilation & Vasoconstriction & $\begin{array}{c}\text { Inhibition of the } \\
\text { renin-angiotensin system }\end{array}$ & Diuresis \\
\hline B-type natriuretic peptide & $\checkmark$ & & $\checkmark$ \\
Atrial natriuretic peptide & $\checkmark$ & & $\checkmark$ \\
Adrenomedullin & $\checkmark$ & $\checkmark$ & $\checkmark$ & \\
Endothelin (1-3) & & & & $\checkmark$ \\
Galanin & $\checkmark$ & & \\
Vasoactive intestinal peptide & $\checkmark$ & $\checkmark$ & & \\
Vasopressin & & $\checkmark$ & & \\
\hline
\end{tabular}

affect proprioception of neck position, and thereby inappropriately trigger a baroreceptor reflex.

Compromise of cerebrovascular and other organ perfusion in a given individual may also occur as a consequence of coexisting disease processes (e.g., structural heart disease and cardiac arrhythmias, diabetes, renal failure), or the adverse effects of prescribed medications. These latter circumstances are very common, and in older individuals are a major source of syncope and OI. Finally, and least frequent but having a worrisome prognosis are progressive diseases of the nervous system (Table 3 ). These conditions are fortunately relatively uncommon causes of syncope or OI in the general population, and are best cared for by neurologists.

\section{ANS disturbances in syncope and OI}

The ANS is comprised of a complex network of neural fibers, central connections, chemical transmitters, and enzymes that ultimately control the function of all organ systems [3]. In health, the ANS is responsible for appropriate modulation of critical physiological functions including regulation of respiratory, blood pressure and cardiac inotropic and chronotropic states, body temperature, and gastrointestinal, endocrine and genitourinary status. For the most part, ANS activity operates unnoticed, with its principal arms (i.e., sympathetic and parasympathetic nervous systems) balancing organ function as required with changing metabolic needs. Unfortunately, a number of conditions may undermine normal ANS function (Table 3), and in terms of affecting the CVS, the result may be intermittent hypotension and syncope or other manifestations of presumed ANS-CVS instability that require an excessive and symptom-inducing compensatory response, such as the sinus tachycardia in POTS. Further, given the ANS's universal role in organ system function, a multitude of concomitant symptoms associated with a variety of organs should not be a surprise. Thus syncope associated with chronic or temporary ANS dysfunction is often accompanied at some stage by abnormal temperature regulation, disturbances of hearing, disturbed respiratory and cardiac rate, gastrointestinal upset, a lightheaded sensation and fatigue. 
Table 3. Principal autonomic disorders (after Mathias et al. [3]).

\begin{tabular}{l}
\hline PRIMARY \\
\hline Chronic autonomic failure syndrome \\
Pure (primary) autonomic failure \\
Multiple system atrophy \\
Autonomic failure with Parkinson's disease \\
\hline SECONDARY \\
\hline Metabolic diseases \\
Diabetes mellitus \\
Chronic renal failure \\
Chronic liver disease \\
Alcoholic toxicity \\
Inflammatory disease \\
Guillain-Barre syndrome \\
Transverse myelitis \\
Autosomal recessive trait \\
Familial dysautonomia (Riley-Day) \\
Dopamine beta-hydroxylase deficiency \\
Autosomal dominant \\
Familial amyloid neuropathy \\
Trauma \\
Spinal cord trauma \\
Sestural tachycardia syndrome
\end{tabular}

While temporary functional disturbances of ANS-CVS interaction account for most forms of syncope, other less common ANS morbidities should not be overlooked (Table 3 ). These include Parkinson's disease, pure autonomic failure and multiple system atrophy. Additionally, uncommon inflammatory conditions, such as Guillan-Barré, may merit consideration. In all of these cases, the essential pathophysiology is transient failure of blood pressure control resulting in self-limited cerebral hypo-perfusion that cannot be fully compensated for by cerebrovascular auto-regulation [4].

As a group, conditions causing disturbances of ANS function may markedly limit an affected individual's ability to carry out activities of daily living and thereby undermine quality-of-life, and may result in substantial economic loss due to loss of employment and cost of care. In many instances, particularly in older patients susceptible to orthostatic hypotension $(\mathrm{OH})$, ANS dysfunction is also associated with increased morbidity and mortality, due primarily to greater potential for falls and injury.

\section{Neuropeptides as potential diagnostic tools in syncope evaluation}

A number of groups have studied the usefulness of common cardiac biomarkers in the diagnostic and prognostic assessment in patients with syncope. The rationale for use of NPs is primarily that they may be elevated in syncope patients for an extended period of time after symptoms have resolved; this would be expected particularly in individuals with cardiovascular disease and impaired hemodynamics, and might thereby suggest an arrhythmic or structural cardiac disorder as the basis for symptoms.

Only a few studies have to date assessed the potential value of NPs to help identify the CVS origin for syncope or near syncope. For instance, Tanimoto et al. [6] examined retrospectively the usefulness of B-type natriuretic peptide (BNP) as a marker for distinguishing cardiac from non-cardiac causes of syncope. They evaluated BNP concentrations in 148 consecutive syncope patients. The 'gold standard' for cardiac syncope was deemed to be evidence for one of the following conditions: aortic stenosis, hypertrophic cardiomyopathy, pulmonary embolism, cardiac tamponade, ventricular tachycardia, or bradycardia. A BNP cut-off value of $40 \mathrm{pg} / \mathrm{mL}$ and above was used to assign a cardiac cause of syncope; they reported that the sensitivity and specificity values for identification of cardiac syncope were $82 \%$ and $92 \%$, respectively (Fig. 2).

Unfortunately, the study by Tanimoto et al. [6] has a number of important limitations. Most importantly, as is evident from Figure 2 modified from their publication, the overlap between cardiac and non-cardiac causes is too great to provide a useful clinical test. Perhaps a $\mathrm{BNP} \geq 75 \mathrm{pg} / \mathrm{mL}$ would have more effectively excluded non-cardiac causes of syncope in their patients. Secondly, being retrospective, the reliability of the original clinical diagnosis is not verifiable. Finally, given the potential seriousness of cardiac syncope, a useful clinical diagnostic screening test must offer much higher sensitivity so as to avoid missing possible cases.

More recently, Pfister et al. [7] proposed $\mathrm{N}$-terminal B-type natriuretic peptide (NT-proBNP) as a prognostic predictor of adverse outcome among syncope patients being evaluated in the Emergency Department (ED). NT-proBNP levels were significantly higher in patients with cardiac compared to non-cardiac syncope. At a cut-off value of $156 \mathrm{pg} / \mathrm{mL}$, NT-proBNP showed a sensitivity of 


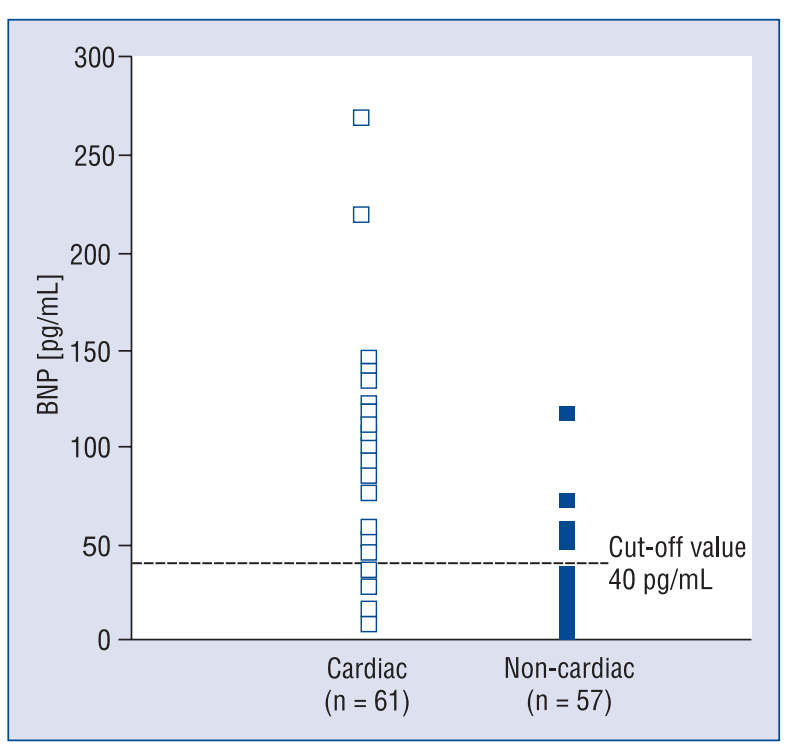

Figure 2. Findings comparing B-type natriuretic peptide (BNP) values in patients deemed to have cardiac and non-cardiac causes of syncope. Note the substantial overlap of values. See text for discussion. The graph has been modified after Tanimoto et al. [6].

$89.7 \%$, a specificity of $51.8 \%$ and a negative predictive value of $84.3 \%$ for the diagnosis of cardiac syncope. Increasing NT-proBNP was a significant predictor of cardiac syncope (OR 3.7, 95\% CI 2.3-5.8 per standard deviation of Log NT-proBNP, $\mathrm{p}<0.001$ ) and addition of NT-proBNP significantly improved a predictive model including heart rate, history of structural heart disease and abnormal electrocardiogram. An important criticism of this evaluation is that the study was performed on hospitalized patients with the results being directly translated to a risk stratification approach of the newly arriving patient in the ED.

In summary, the role of NPs, particularly BNP and NT-proBNP diagnostic screening in the ED or urgent care environment is appealing but the findings to date are unconvincing. Currently, patients in whom an explanation for syncope is not found during the initial evaluation and who are judged as being at increased risk are hospitalized and undergo a time and resource-consuming diagnostic procedure. There is clearly a need for improved diagnostic capabilities and clinical parameters in a triage setting. Biomarkers such as BNP and NT-proBNP, which are available widely as a commercial assay, could provide a low cost tool for risk stratification. Further evaluation is needed.

\section{Neuropeptides and peptide hormones in the pathophysiology of reflex syncope}

Reflex syncope refers to a heterogeneous group of conditions in which cardiovascular reflexes, that normally act to stabilize the circulation and allow it to respond to conditions of stress or exertion, become transiently inappropriate. The triggers are sometimes clear-cut (e.g., pain, emotional upset, micturition), but often that is not the case. The adverse outcome is typically veno- and vaso-dilatation and marked or relative bradycardia. If sufficiently severe, arterial blood pressure may fall, and global cerebral hypo-perfusion may cause syncope. Additionally, as noted earlier, multiple organ systems other than the CVS are affected, and gastrointestinal, respiratory and constitutional (e.g., fatigue) symptoms commonly accompany the hypotension.

Vasovagal syncope (VVS) is the most common of the group of reflex (neurally-mediated) syncope conditions, and several studies have assessed neurohumoral changes accompanying VVS by use of head-up tilt-table testing. In this regard, changes in circulatory catecholamines have tended to be of greatest interest given the apparent association of VVS with anxiety or stress states [8-14]. However, the potential role of NPs has also attracted attention; specifically, BNP [8], vasopressin (VP) $[8,15,16]$, atrial natriuretic peptide (ANP) $[8,17]$, adrenomedullin (ADM) [17-19], galanin (GAL) $[19,20]$, and endothelin-1 (ET-1) [17, 21-23]. A summary of the primary physiologic effects of these NPs is shown in Table 2.

\section{Vasopressin}

Vasopressin was among the first NPs (or 'peptide hormones') to be evaluated as a potential contributor to VVS pathophysiology $[8,15,16]$. All studies agree that at the time of tilt-table induced reflex syncope VP levels are elevated. By way of example, Jardine et al. [8] observed a statistically significant VP increase 5 min before symptoms, with an even greater excess occurring at the time of syncope. However, in other reports an increase in VP levels occurred during upright tilt in both subjects who eventually develop syncope and those who do not faint [15]. VP has also been reported to exhibit a biphasic increase: first at 3 to $7 \mathrm{~min}$ of upright tilt in both normal controls and those who faint, and again at $45 \mathrm{~min}$ in controls and at the time of syncope in those who faint [15]. 
In any event, the manner in which VP might enhance VVS susceptibility is unclear. Jardine et al. [8] hypothesized that VPs direct pressor effect may be overcome by an indirect effect enhancing arterial and ventricular mechanoreceptor activity; however, other reports suggest that carotid baroreceptor activity is down-regulated by VP. Clearly, further study of VP-baroreceptor interaction is needed.

\section{ANP and BNP}

Jardine et al. [8] examined BNP and ANP levels during head-up tilt testing in control subjects and individuals who developed reflex syncope. BNP did not differ appreciably in the two patient groups and consequently is not likely to play a pathophysiological role. ANP levels tended to be higher throughout the study in syncope-prone patients compared to controls. However, in both groups, ANP values fell slowly and comparably during the period of extended upright posture, but were not appreciably altered by whether syncope occurred or not.

Fedorowski et al. [17] recently examined the relationships between certain NPs and mechanisms underlying syncope in a series of patients with unexplained transient loss of consciousness undergoing head-up tilt table testing; a cardiac etiology for symptoms was deemed excluded by the initial clinical evaluation. They studied the mid-regional fragments of pro-atrial natriuretic peptide (MR-proANP); findings revealed that ANP levels tended to be lowest in patients prone to tilt-induced fainting and highest in non-fainters (Fig. 3). Taken together, the observations of Jardine et al. [8] and Fedorowski et al. [17] may be reasonably interpreted to reflect the impact of progressively diminished atrial stretch during upright posture, with NP's acting to provide a fluid conservation state particularly in syncope-prone individuals.

\section{Galanin}

Galanin (GAL) is a neuropeptide that is widely distributed in the central and peripheral nervous systems. GAL interacts with both sympathetic and vagal systems as well as with neurotransmitters, such as serotonin. In animals, GAL can lower blood pressure and attenuate vagally-induced slowing of the heart rate. In humans, the administration of GAL depresses basal norepinephrine and norepinephrine responses to both assumption of upright posture and insulin-induced hypoglycemia.

Bondanelli et al. [19] examined plasma GAL changes during head-up tilt test (HUTT) in healthy subjects and patients with recurrent VVS. In

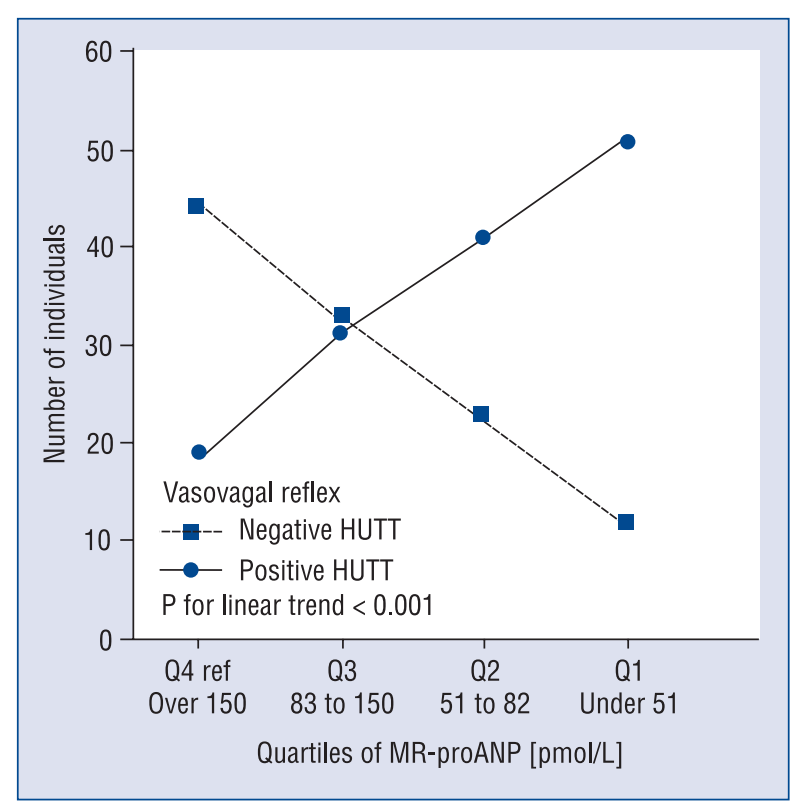

Figure 3. Findings illustrating the inverse relationship between atrial natriuretic peptide (ANP) values and tendency to positive head-up tilt test (HUTT) result in patients with vasovagal syncope. The ordinate indicates the number of subjects with a positive (solid line) or negative (dashed line) HUTT. The abscissa depicts neuropeptides values by quartile range with highest values at the left. The largest number of positive HUTT's tended to be associated with low ANP values. Conversely, patients with negative HUTT tended to have highest ANP levels; MR-proANP - mid-regional fragments of pro-ANP. Graph modified after Fedorowski et al. [17].

healthy control subjects, GAL did not change during HUTT. However, GAL levels increased in VVS patients who did not develop syncope (negative HUTT), whereas it remained unchanged in VVS patients who developed syncope (positive HUTT).

Plasek et al. [20] obtained slightly different results from the Bondanelli et al. study [19]. GAL increase differentiated cases from controls better than it differentiated positive and negative responses to HUTT, i.e. there was an increase in plasma GAL for VVS patients during HUTT, regardless of whether they developed syncope or not during the test. Overall despite the controversies on the role of GAL in VVS, GAL might be useful as a marker of VVS recurrence.

\section{Endothelin}

Kaufmann et al. [22] and White et al. [23] provided early observations on the association between plasma endothelin levels and outcomes during upright tilt. Kaufmann et al. [22] noted that 
plasma endothelin (and VP) rose in VVS patients; this did not occur in autonomic failure patients. Conversely, White et al. [23] did not observe any increase in ET-1 among VVS susceptible subjects, but did in non-fainters. More recently, Fedorowski et al. [17] re-assessed the relationships between C-terminal endothelin-1 precursor fragment (CT-proET-1) and the main mechanisms underlying syncope diagnosed during head-up tilt in a series of patients with unexplained transient loss of consciousness. Consistent with the observations of White et al. [23], in neurally-mediated syncope, ET-1 production was down-regulated.

\section{Neuropeptides in postural tachycardia syndrome}

Postural tachycardia syndrome is principally characterized by excessive sinus tachycardia when the affected individual is in the upright position; however, it is frequently also accompanied by numerous other symptoms (e.g., dizziness, fatigue, exercise intolerance, etc.). POTS remains a condition that has proved clinically very challenging. Little is known of the potential role of vasoactive NPs in this condition, except for one study examining adrenomedullin $(\mathrm{ADM})$ levels in children with presumed POTS.

\section{Adrenomedullin}

Adrenomedullin is a vasodilator (acting through nitric oxide) and a natriuretic agent that increases glomerular filtration. It is widely accepted that measurement of the concentration of a more stable mid-regional fragment of pro-ADM (MR-proADM) is a suitable surrogate for ADM concentration.

Zhang et al. [18] examined the predictive value of the MR-proADM in assessing the therapeutic efficacy of midodrine hydrochloride for children with POTS. The authors reported higher levels of MR-proADM in otherwise healthy children with a clinical diagnosis of POTS compared with normal controls. Further, they concluded that MR-proADM $>61.5 \mathrm{pg} / \mathrm{mL}$ provided a useful cut-off to predict effectiveness of therapy with midodrine. Finally they observed that the higher the ADM level, the greater the midodrine effectiveness; the finding is at best counter-intuitive.

In an editorial [10] accompanying the article by Zhang et al. [18], concerns were raised regarding the study design, the midodrine dose employed, and the physiological mechanism for the apparent efficacy of midodrine in patients with elevated ADM. Excluding concerns related to the utility of midodrine in POTS patients, the possibility that vasoactive peptides, particularly $\mathrm{ADM}$, contribute to the pathophysiology of POTS is intriguing. Even if NPs are epiphenomena in POTS, the study from Zhang et al. [18] raises the prospect of utilizing vasoactive peptide concentrations as useful quantitative markers of POTS severity, and potentially as a means other than symptom status that can be tracked in individual patients as treatments are introduced.

\section{Neuropeptides in $\mathrm{OH} /$ syncope}

The term 'orthostatic hypotension' describes a condition in which the ANS fails to maintain a stable blood pressure in the face of orthostatic stress. $\mathrm{OH}$ is generally defined as a reduction in a systolic blood pressure of at least $20 \mathrm{~mm} \mathrm{Hg}$ or diastolic blood pressure of at least $10 \mathrm{~mm} \mathrm{Hg}$ with 3 min of standing [1]. However, a smaller fall in blood pressure that is associated with symptoms can be of equal importance, as can a slow but steady decline in blood pressure over a somewhat longer period (10-15 $\mathrm{min})$.

A variety of conditions can result in failure of orthostatic blood pressure control; these causes are often classified into primary and secondary. Primary causes of $\mathrm{OH}$ are for the most part idiopathic, whereas secondary forms are usually associated with a particular disease (such as diabetes), pharmacotherapy (e.g., diuretics, vasodilators), or a known biochemical or structural abnormality. The latter conditions include pure autonomic failure characterized by widespread evidence of autonomic failure as well as $\mathrm{OH}$, but with no other neurologic involvement, and multiple system atrophy (once known as Shy-Drager syndrome), a sporadic, progressive and ultimately fatal disorder characterized by OI and widespread evidence of ANS dysfunction together with manifestations of Parkinsonism and cerebellar ataxia, alone or in combination [3]. Some patients with classic Parkinson disease may also develop signs of ANS failure, including $\mathrm{OH}$.

A number of pharmacologic agents may produce or exacerbate $\mathrm{OH}$. These agents include tricyclic antidepressants, ethanol, angiotensin-converting enzyme inhibitors, alpha-receptor blockers, calcium channel blockers, phenothiazines, opiates, bromocriptine, and beta-blockers. Therapy includes increasing dietary fluid and salt intake, exercise, and compression garments in addition to discontinuing the use of offending agents, if possible. Volume expanders such as fludrocortisone acetate and vasoconstrictors such as midodrine hydrochloride may be useful in selected patients. 
In most cases, careful clinical evaluation is usually able to identify a likely etiology for $\mathrm{OH}$; however, in approximately $20-40 \%$ of cases the cause is unknown. Extrinsic pharmacologic agents (e.g., diuretics and vasodilators) are well-known causes of $\mathrm{OH}$ as noted above; however the possibility exists that intrinsic neurohumoral agents with similar properties may be a contributing factor was initially suggested by observations in certain paraneoplastic syndromes; specifically, findings in small cell lung cancer in which elevated ANP was associated with $\mathrm{OH}[24]$.

Several intrinsic NPs including BNP and NT-proBNP, ANP, and ADM exhibit diuretic and vasodilator properties. Although these NPs are usually only elevated in volume overload states, excess NP related to tumors or inflammatory conditions has been previously observed, and associated with $\mathrm{OH}$ in some cases.

\section{ANP and BNP}

As noted above, Fedorowski et al. [17] assessed several NPs in various forms of syncope. ANP levels were not markedly altered in $\mathrm{OH}$. On the other hand, with respect to BNP, we recently noted correspondence between $\mathrm{OH}$ symptoms and NT-proBNP levels in euvolemic patients without other $\mathrm{OH}$ explanation; most had a prior history of solid organ transplants or inflammatory disease. In healthy subjects, plasma BNP levels are low $(<300 \mathrm{pg} / \mathrm{mL})$. The major stimulus for BNP release is mechanical myocyte stress usually due to ventricular volume and/or pressure overload as may occur in hypervolemic systolic heart failure [25]. However, apart from renal excretion other factors such as marked obesity and inflammation may modify circulating BNP and NT-proBNP concentrations [26]. Obesity lowers pro-BNP, and inflammation through its effects on cytokines increases NT-proBNP, albeit modestly and not to the levels noted in our cohort $[27,28]$. Serum levels of NT-proBNP have been associated with allograft function in recipients of renal transplants [29]. Median NT-proBNP in renal transplant patients reach levels of $500-600 \mathrm{pg} / \mathrm{mL}$ in patients with estimated glomerular filtration rate $>60 \mathrm{~mL} / \mathrm{min}$. Levels are typically elevated in patients with delayed graft function and hypervolemia. In the same study, the type of immunosuppression did not play a role in NT-proBNP levels. Our group of renal transplant patients did not have delayed graft function dysfunction, and were euvolemic or hypovolemic on presentation.

In heart failure, the known natriuretic, diuretic and vasorelaxant properties of BNP induce an increase in effective renal plasma flow, glomerular filtration rate, urine flow rate, and sodium excretion. ANP has similarly been shown to inhibit postural release of renin and VP in healthy humans. The increase in NP levels has been considered to represent an intrinsic compensatory response to volume excess. Prolonged BNP release in the absence of volume overload, as has been reported with certain malignancies might be expected to trigger $\mathrm{OH}$. Potential extracardiac sources of BNP secretion include the central nervous system, lung, thyroid, adrenal glands, kidney, spleen, small intestine, ovary, uterus and striated muscle. To date, however, there are no reports of non-cardiac sources resulting in sustained high NT-proBNP levels comparable to those observed in our patients.

\section{Vasopressin}

Vasopressin appears to play a role in moderating any tendency to hypotension induced by upright posture. Head-up posture should be associated with increased VP levels, thereby providing a positive pressor response [30]. Further, the appropriateness of VP response to upright posture may serve as a means of assessing the integrity of central baroreflex arcs.

\section{Endothelin-1}

In $\mathrm{OH}$, Fedorowski et al. [17] observed hyperactivity of the endothelin system. They reported a strong linear relationship between increasing supine CT-proET-1 levels and orthostatic instability (Fig. 4). Since ET-1 is one of the most potent vasoconstrictors, producing a sustained increase in blood pressure, up-regulation of ET-1 production may be a way to compensate for a hypotensive tendency during orthostasis. Suppression of the endothelin system was highly suggestive of a cardioinhibitory reflex. Therefore, endothelin level may suggest a diagnosis of either 'pure' vasovagal reflex (low concentration) or $\mathrm{OH}$ (high concentration), the latter indicating a tendency towards dominant vasodilation.

\section{Conclusions}

A wide range of NPs and PHs have been identified, and many exert cardio-active effects that could in theory prove helpful in identifying causes of syncope, or even explaining the pathophysiology of certain syncope syndromes. To date, the principal NP/PHs that have been studied in this context are: ANP, ADM, BNP, ET-1, GAL, and VP. Findings are as yet not definitive. However, the intrinsic vasoconstrictor ET-1 may be elevated in some $\mathrm{OH}$ 


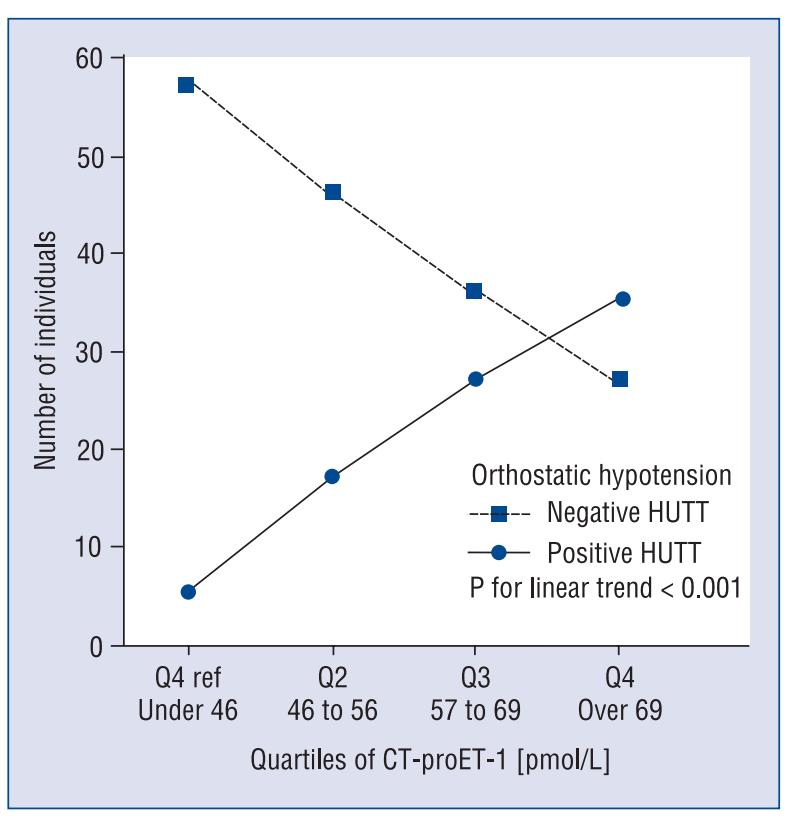

Figure 4. Findings illustrating the direct relationship between endothelin-1 (ET-1) values and tendency to positive head-up tilt test (HUTT) result in patients with orthostatic syncope. The ordinate indicates the number of subjects with a positive (solid line) or negative (dashed line) HUTT. The highest ET-1 levels are at the right on the abscissa. A positive orthostatic response on HUTT tended to be associated with the highest ET-1 values suggesting a possible compensatory response; CT-proET-1 - C-terminal ET-1 precursor fragment. Graph modified after Fedorowski et al. [17].

cases presumably in an attempt to compensate for vasodilation and hypotension of other etiologies. As such ET-1 may be a useful marker for $\mathrm{OH}$. Conversely, elevated NT-proBNP may play a role in causing vasodilation and hypotension in some forms of $\mathrm{OH}$ of unknown cause, and may be a marker in other patients of a cardiovascular cause of syncope. The contributions of other NPs are even more uncertain. In the end, the study of NP/PHs in syncope and OI syndromes has just begun, and considerable further future effort will be needed to clarify their possible contributions to these conditions.

\section{Acknowledgements}

We would like to acknowledge Barry L.S. Detloff for his assistance in compiling the Figures and Tables.

Dr. Benditt was supported in part by a philanthropic gift to the University of Minnesota Foundation in support of Heart-Brain Research by the Dr. Earl E. Bakken Family.

Conflict of interest: None declared

\section{References}

1. Task Force for the Diagnosis and Management of Syncope, European Society of Cardiology (ESC), European Heart Rhythm Association (EHRA) et al. Guidelines for the diagnosis and management of syncope (version 2009). Eur Heart J, 2009; 30: 2631-2671.

2. Brignole M, Menozzi C, Bartoletti A et al. A new management of syncope: Prospective systematic guideline-based evaluation of patients referred urgently to general hospitals. Eur Heart J, 2006; 27: 76-82.

3. Mathias CJ. Orthostatic hypotension: causes, mechanisms, and influencing factors. Neurology, 1995; 45: S6-S11.

4. Schondorf R, Benoit J, Stein R. Cerebral autoregulation is preserved in postural tachycardia syndrome. J Appl Physiol, 2005; 99: 828-835.

5. Ejaz AA, Haley WE, Wasiluk A, Meschia JF, Fitzpatrick PM. Characteristics of 100 consecutive patients presenting with orthostatic hypotension. Mayo Clin Proc, 2004; 79: 890-894.

6. Tanimoto K, Yukiiri K, Mizushige $\mathrm{K}$ et al. Usefulness of brain natriuretic peptide as a marker for separating cardiac and noncardiac causes of syncope. Am J Cardiol, 2004; 93: 228-230.

7. Pfister R, Diedrichs H, Larbig R, Erdmann E, Schneider CA. NT-pro-BNP for differential diagnosis in patients with syncope. Int J Cardiol, 2009; 133: 51-54.

8. Jardine DL, Melton IC, Crozier IG, Bennett SI, Donald RA, Ikram H. Neurohormonal response to head-up tilt and its role in vasovagal syncope. Am J Cardiol, 1997; 79: 1302-1306.

9. Sander-Jensen K, Secher NH, Astrup A et al. Hypotension induced by passive head-up tilt: endocrine and circulatory mechanisms. Am J Physiol, 1986; 251: R742-R748.

10. Benditt DG, Chen LY. Peptides in postural orthostatic tachycardia syndrome: Players or epiphenomena? J Am Coll Cardiol, 2012; 60: 321-323.

11. Benditt DG, Detloff BL, Adkisson WO et al. Age-dependence of relative change in circulating epinephrine and norepinephrine concentrations during tilt-induced vasovagal syncope. Heart Rhythm, 2012; 9: 1847-1852.

12. Ermis C, Samniah N, Sakaguchi S et al. Comparison of catecholamine response during tilt-table-induced vasovagal syncope in patients 65 years of age. Am J Cardiol, 2004; 93: 225-227.

13. Ermis C, Samniah N, Lurie KG, Sakaguchi S, Benditt DG. Adre$\mathrm{nal} /$ renal contribution to circulating norepinephrine in posturally induced neurally mediated reflex syncope. Am J Cardiol, 2003; 91: 746-750.

14. Benditt DG, Ermis C, Padanilam B, Samniah N, Sakaguchi S. Catecholamine response during haemodynamically stable upright posture in individuals with and without tilt-table induced vasovagal syncope. Europace, 2003; 5: 65-70.

15. Roul G, Riehl-Aleil V, Germain P, Bareiss P. Neurohormonal profile before and after beta-blockade in patients with neurocardiogenic syncope. Pacing Clin Electrophysiol, 1999; 22: 1020-1030.

16. Liard JF. Vasopressin in cardiovascular control: role of circulating vasopressin. Clin Sci (Lond), 1984; 67: 473-481.

17. Fedorowski A, Burri P, Struck J, Juul-Moller S, Melander O. Novel cardiovascular biomarkers in unexplained syncopal attacks: The SYSTEMA cohort. J Intern Med, 2013; 273: 359-367.

18. Zhang F, Li X, Ochs $\mathrm{T}$ et al. Midregional pro-adrenomedullin as a predictor for therapeutic response to midodrine hydrochloride in children with postural orthostatic tachycardia syndrome. J Am Coll Cardiol, 2012; 60: 315-320. 
19. Bondanelli M, Alboni P, Margutti A et al. Plasma galanin response to head-up tilt in normal subjects and patients with recurrent vasovagal syncope. Metabolism, 2003; 52: 315-321.

20. Plasek J, Doupal V, Furstova J et al. The role of adrenomedullin and galanin in recurrent vasovagal syncope: A case control study. Biomed Pap Med Fac Univ Palacky Olomouc Czech Repub, 2013; 157: 162-167.

21. Magerkurth C, Riedel A, Braune S. Permanent increase in endothelin serum levels in vasovagal syncope. Clin Auton Res, 2005; 15: 299-301.

22. Kaufmann H, Oribe E, Oliver JA. Plasma endothelin during upright tilt: relevance for orthostatic hypotension? Lancet, 1991; 338: 1542-1545.

23. White M, Cernacek P, Courtemanche M et al. Impaired endothelin-1 release in tilt-induced syncope. Am J Cardiol, 1998; 81: 460-464.

24. Campling BG, Sarda IR, Baer KA et al. Secretion of atrial natriuretic peptide and vasopressin by small cell lung cancer. Cancer, 1995; 75: 2442-2451.
25. Yoshimura M, Yasue H, Okumura K et al. Different secretion patterns of atrial natriuretic peptide and brain natriuretic peptide in patients with congestive heart failure. Circulation, 1993; 87: 464-469.

26. Baggish AL, van Kimmenade RR, Januzzi JL, Jr. The differential diagnosis of an elevated amino-terminal pro-B-type natriuretic peptide level. Am J Cardiol, 2008; 101: 43-48.

27. Chua G, Kang-Hoe L. Marked elevations in N-terminal brain natriuretic peptide levels in septic shock. Crit Care, 2004; 8: R248-R250.

28. Chung CP, Solus JF, Oeser A et al. N-terminal pro-brain natriuretic peptide in systemic lupus erythematosus: relationship with inflammation, augmentation index, and coronary calcification. J Rheumatol, 2008; 35: 1314-1319.

29. Bodlaj G, Hubmann R, Saleh K et al. Serum levels of N-terminal pro-B-type natriuretic peptide are associated with allograft function in recipients of renal transplants. Wien Klin Wochenschr, 2009; 121: 631-637.

30. Zerbe RL, Henry DP, Robertson GL. Vasopressin response to orthostatic hypotension. Etiologic and clinical implications. Am J Med, 1983; 74: 265-271. 